Теорія Ймовір. та Матем. Статист. Вип. 77, 2007
Theor. Probability and Math. Statist.

No. 77, 2008, Pages 1-13

S 0094-9000(08)00743-6

Article electronically published on January 14, 2009

\title{
RUIN PROBABILITY FOR AN INSURER INVESTING IN SEVERAL RISKY ASSETS
}

UDC 519.21

\author{
M. V. BRATYK
}

\begin{abstract}
The ruin probability of an insurer is studied for the classical CramérLundberg model with finite exponential moments. The nonclassical property of the model considered in the paper is the possibility to invest in two different risky assets (which may be dependent) whose price processes are either described by geometric Brownian motions or are semimartingales with absolutely continuous characteristics with respect to Lebesgue measure. We study the ruin probability for the case where a free credit is not available in the money market and where the insurer can invest in a finite number of risky assets whose price processes are described by jointly independent Brownian motions.
\end{abstract}

\section{INTRODUCTION}

An optimal strategy and the ruin probability are obtained in the paper [1 for an insurer whose activity is described by the Cramér-Lundberg model for which the insurer is allowed to invest in a single asset.

An estimate of the ruin probability is obtained in [2] for the case where an insurer invests in a single risky asset modelled by a semimartingale possessing absolutely continuous characteristics with respect to Lebesgue measure.

Both papers mentioned above deal with the case where the insurer is allowed to invest in a single risky asset only. Section 2 below generalizes a result obtained in the paper [1] for the case where an insurer is allowed to invest in two (possibly dependent) risky assets. An estimate of the ruin probability is found in Section 3 for the same setting as that considered in Section 2 of the paper [2].

\section{THE RUIN PROBABILITY AND OPTIMAL INVESTMENT STRATEGY FOR AN INSURER INVESTING IN CORRELATED RISKY ASSETS}

2.1. The model. Let the capital of an insurer at the time $t \geq 0$ be described by the equation

$$
Y\left(t, x, k_{1}, k_{2}\right)=x+c t-\sum_{i=1}^{N(t)} X_{i}+\left(\frac{k_{1}}{S_{1}} \cdot S_{1}\right)(t)+\left(\frac{k_{2}}{S_{2}} \cdot S_{2}\right)(t)
$$

where $x \geq 0$ is the initial capital, $X_{i}$ is a sequence of independent identically distributed random variables (copies of a random variable $X$ ), $N(t)$ is a Poisson process having intensity $\lambda$ and independent of $X_{i}$, and where $c \in \mathbb{R}$ is a premium rate. The insurer

2000 Mathematics Subject Classification. Primary 60G44, 60H30; Secondary 62P05, 60K10.

Key words and phrases. Cramér-Lundberg model, ruin probability, investment strategy, geometric Brownian motion. 
is allowed to invest in two different risky assets whose price processes are modelled by geometric Brownian motions

$$
d S_{i}(t)=S_{i}(t)\left(a_{i} d t+b_{i} d w_{i}(t)\right), \quad i=1,2,
$$

where $a_{i}, b_{i} \in \mathbb{R}$ are fixed and where $w_{1}(t)$ and $w_{2}(t)$ are Wiener processes with respect to the filtration $F=\left(F_{t}\right)_{t>0}$ generated by the processes $\sum_{i=1}^{N(t)} X_{i}, S_{1}(t)$, and $S_{2}(t)$. We also assume that $\left(w_{1}(t), w_{2}(t)\right)$ is a two-dimensional Gaussian process. Note that we do not assume that the processes $w_{1}(t)$ and $w_{2}(t)$ are independent. Let $\operatorname{cov}\left(w_{1}(t), w_{2}(t)\right)=\sigma t$ for some $-1<\sigma<1$.

Here $k_{1}(t)$ and $k_{2}(t)$ are the processes describing the fractions of the capital invested in the corresponding assets at time $t$,

$$
\left(\frac{k_{i}}{S_{i}} \cdot S_{i}\right)(t)=\left(k_{i} \cdot w_{a_{i}, b_{i}}^{i}\right)(t),
$$

where $w_{a_{i}, b_{i}}^{i}(t)$ is the generalized Wiener process, $w_{a_{i}, b_{i}}^{i}(t)=a_{i} t+b_{i} w_{i}(t)$, and where $\left(k_{i} \cdot w_{a_{i}, b_{i}}^{i}\right)$ means the stochastic integral of the process $k_{i}$ with respect to the process $w_{a_{i}, b_{i}}^{i}(t), i=1,2$.

In what follows we assume that $w_{1}(t)$ and $w_{2}(t)$ are jointly independent of the sequence $X_{i}, i \geq 1$, and process $N(t)$.

Let $h(r)=\mathrm{E} e^{r X}-1$. We assume that there exists a number $r_{\infty} \in(0, \infty)$ such that $h(r)<\infty$ for all $r<r_{\infty}$ and that $h(r) \rightarrow \infty$ as $r \rightarrow r_{\infty}$ (this is the classical assumption). The function $h(r)$ is increasing, concave, continuous on $\left[0, r_{\infty}\right)$, and such that $h(0)=0$.

Let

$$
\Psi\left(x, k_{1}, k_{2}\right)=\mathrm{P}\left\{Y\left(t, x, k_{1}, k_{2}\right)<0 \text { for some } t \geq 0\right\}
$$

be the ruin probability for an insurer. The ruin probability depends on the initial capital $x$ and on the investment strategy $\left(k_{1}, k_{2}\right)$. Let $\tau\left(x, k_{1}, k_{2}\right)=\inf \left\{t: Y\left(t, x, k_{1}, k_{2}\right)<0\right\}$ be the ruin moment.

The set $K$ of admissible strategies is defined by

$$
\begin{aligned}
K=\left\{\left(k_{1}(t), k_{2}(t)\right)_{t \geq 0}:\right. & k_{1}, k_{2} \text { are predictable with respect to the filtration } F, \\
& \left.\mathrm{P}\left\{\int_{0}^{t}\left(k_{i}(s)\right)^{2} d s<\infty\right\}=1, i=1,2 \text {, for all } t \in[0, \infty)\right\} .
\end{aligned}
$$

Put $\Psi^{*}(x)=\inf _{\left(k_{1}, k_{2}\right) \in K} \Psi\left(x, k_{1}, k_{2}\right)$.

2.2. The strategy and an estimate of the ruin probability. The ruin probability for the classical Cramér-Lundberg model is estimated with the help of the following inequality:

$$
\Psi(x) \leq e^{-\nu x}
$$

where $\nu$ is a positive root of the equation $\lambda h(r)=c r$ for $c>\lambda \mathrm{E}\left[X_{1}\right]$. The latter result is called the Lundberg inequality and $\nu$ is called the Lundberg coefficient.

The paper [1] deals with the model where an insurer invests in a single risky asset modelled by a geometric Brownian motion $d S(t)=S(t)(a d t+b d w(t))$ where $a, b \in \mathbb{R}$ are fixed constants. In this case, the ruin probability is estimated as follows:

$$
\Psi^{*}(x) \leq e^{-r_{1} x},
$$

where $r_{1}$ is a positive solution of the equation $\lambda h(r)=c r+a^{2} /\left(2 b^{2}\right)$. Note that $r_{1}$ is greater than the Lundberg coefficient $\nu$.

Below is the main result of the first part of this paper. 
Theorem 2.1. Assume that $b_{i} \neq 0, i=1,2$, for the model described by equation (11). Then the ruin probability $\Psi^{*}(x)$ of an insurer is estimated by

$$
\Psi^{*}(x) \leq e^{-\tilde{r} x},
$$

where $0<\widetilde{r}<r_{\infty}$ is a positive root of the equation

$$
\lambda h(r)=c r+\frac{1}{2} \cdot \frac{\left(\frac{a_{1}^{2}}{b_{1}^{2}}+\frac{a_{2}^{2}}{b_{2}^{2}}-\frac{\sigma a_{1} a_{2}}{b_{1} b_{2}}\right)^{2}}{a_{1}^{2}\left(\frac{1}{b_{1}}-\frac{\sigma}{b_{2}}\right)^{2}+a_{2}^{2}\left(\frac{1}{b_{2}}-\frac{\sigma}{b_{1}}\right)^{2}+2 \sigma a_{1} a_{2}\left(\frac{1}{b_{1}}-\frac{\sigma}{b_{2}}\right)\left(\frac{1}{b_{2}}-\frac{\sigma}{b_{1}}\right)} .
$$

This result follows from Theorem 2.2 below.

We will use the following process. For fixed numbers $x \geq 0$ and $r \geq 0$ and admissible strategy $\left(k_{1}, k_{2}\right) \in K$, let

$$
M\left(t, x, k_{1}, k_{2}, r\right)=e^{-r Y\left(t, x, k_{1}, k_{2}\right)} .
$$

We show that the ruin probability admits the estimate $\Psi\left(x, k_{1}, k_{2}\right) \leq e^{-r x}$ provided that $M\left(t, x, k_{1}, k_{2}, r\right)$ is an $F_{t}$-martingale.

First we find conditions for the process $M\left(t, x, k_{1}, k_{2}, r\right)$ to be an $F_{t}$-martingale. Note that

$$
\begin{aligned}
M\left(t, x, k_{1}, k_{2}, r\right)=\exp \left(-r\left(x+c t-\sum_{i=1}^{N(t)} X_{i}+\int_{0}^{t}\right.\right. & k_{1}(s)\left(a_{1} d s+b_{1} d w_{1}(s)\right) \\
& \left.\left.+\int_{0}^{t} k_{2}(s)\left(a_{2} d s+b_{2} d w_{2}(s)\right)\right)\right) .
\end{aligned}
$$

The stochastic integrals of the processes $k_{i}$ with respect to the processes $w_{i}$ are well defined in view of the inclusion $\left(k_{1}, k_{2}\right) \in K$.

First we choose a strategy $\left(k_{1}, k_{2}\right)$ to satisfy the condition

$$
\mathrm{E} M\left(t, 0, k_{1}, k_{2}, r\right)=1 \text {. }
$$

We have

$$
\begin{aligned}
& \mathrm{E} M(\left.t, 0, k_{1}, k_{2}, r\right) \\
&=\mathrm{E} \exp \left(-r\left(c t-\sum_{i=1}^{N(t)} X_{i}+a_{1} \int_{0}^{t} k_{1}(s) d s+b_{1} \int_{0}^{t} k_{1}(s) d w_{1}(s)\right.\right.\left.\left.+a_{2} \int_{0}^{t} k_{2}(s) d s+b_{2} \int_{0}^{t} k_{2}(s) d w_{2}(s)\right)\right) \\
&=\mathrm{E}\left[\exp \left(-r c t+r a_{1} \int_{0}^{t} k_{1}(s) d s+r a_{2} \int_{0}^{t} k_{2}(s) d s\right) e^{h(r) \lambda t}\right. \\
&\left.\quad \times \exp \left(\frac{r^{2} b_{1}^{2}}{2} \int_{0}^{t} k_{1}^{2}(s) d s+\frac{r^{2} b_{2}^{2}}{2} \int_{0}^{t} k_{2}^{2}(s) d s+r^{2} \sigma b_{1} b_{2} \int_{0}^{t} k_{1}(s) k_{2}(s) d s\right)\right] \\
&=\mathrm{E} \exp \left(\int_{0}^{t} f\left(k_{1}, k_{2}, r, s\right) d s\right),
\end{aligned}
$$

where

$$
\begin{aligned}
& f\left(k_{1}, k_{2}, r, s\right) \\
& \quad=\lambda h(r)-r c-r\left(k_{1}(s) a_{1}+k_{2}(s) a_{2}\right)+\frac{1}{2} r^{2}\left(b_{1}^{2} k_{1}^{2}(s)+b_{2}^{2} k_{2}^{2}(s)+\sigma b_{1} b_{2} k_{1}(s) k_{2}(s)\right) .
\end{aligned}
$$

It is clear that the equality $\mathrm{E} M\left(t, 0, k_{1}, k_{2}, r\right)=1$ holds if $f\left(k_{1}, k_{2}, r, s\right)=0, s \geq 0$. 
Since $\widetilde{r}$ is a root of the equation

$$
\lambda h(r)=r c+r\left(k_{1}(s) a_{1}+k_{2}(s) a_{2}\right)-\frac{1}{2} r^{2}\left(b_{1}^{2} k_{1}^{2}(s)+b_{2}^{2} k_{2}^{2}(s)+\sigma b_{1} b_{2} k_{1}(s) k_{2}(s)\right),
$$

the best possible upper bound $\Psi(x) \leq e^{-\widetilde{r} x}$ corresponds to the maximal root of equation (2). In view of the restrictions posed on the function $h(r)$, the maximal root of equation (2) corresponds to the maximum of the expression

$$
r\left(k_{1} a_{1}(s)+k_{2} a_{2}(s)\right)-\frac{1}{2} r^{2}\left(b_{1}^{2} k_{1}^{2}(s)+b_{2}^{2} k_{2}^{2}(s)+\sigma b_{1} b_{2} k_{2}(s) k_{2}(s)\right) .
$$

Let $k_{1}(s)=\mu(s) k(s)$ and $k_{2}(s)=(1-\mu(s)) k(s)$. Then equation (2) takes the following form:

$$
\begin{aligned}
\lambda h(r)= & c r+r k(s)\left(\mu(s) a_{1}+(1-\mu(s)) a_{2}\right) \\
& -r^{2}(k(s))^{2}\left(\frac{(\mu(s))^{2} b_{1}^{2}}{2}+\frac{(1-\mu(s))^{2} b_{2}^{2}}{2}+\sigma \mu(s)(1-\mu(s)) b_{1} b_{2}\right) .
\end{aligned}
$$

We find the maximum of

$$
\begin{aligned}
& r k(s)\left(\mu(s) a_{1}+(1-\mu(s)) a_{2}\right) \\
& \quad-r^{2}(k(s))^{2}\left(\frac{(\mu(s))^{2} b_{1}^{2}}{2}+\frac{(1-\mu(s))^{2} b_{2}^{2}}{2}+\sigma \mu(s)(1-\mu(s)) b_{1} b_{2}\right) .
\end{aligned}
$$

The latter expression is a second degree polynomial with respect to $r k(s)$. Since the leading coefficient of this polynomial is negative, the maximum of the above expression is attained at

$$
r k(s)=\frac{\mu a_{1}+(1-\mu) a_{2}}{\mu^{2} b_{1}^{2}+(1-\mu)^{2} b_{2}^{2}+2 \sigma \mu(1-\mu) b_{1} b_{2}}
$$

if $\mu(s)=\mu$ is fixed.

Substituting the latter expression for $r k(s)$ in (3) we obtain the equation

$$
\lambda h(r)=c r+\frac{\left(\mu a_{1}+(1-\mu) a_{2}\right)^{2}}{2\left(\mu^{2} b_{1}^{2}+(1-\mu)^{2} b_{2}^{2}+2 \sigma \mu(1-\mu) b_{1} b_{2}\right)} .
$$

Maximizing the second term on the right hand side of equation (5) with respect to $\mu$ we see that the maximum is attained at the point

$$
\mu(s)=\mu_{*}=\frac{\frac{a_{1}}{b_{1}^{2}}-\frac{\sigma a_{1}}{b_{1} b_{2}}}{\frac{a_{1}}{b_{1}^{2}}+\frac{a_{2}}{b_{2}^{2}}-\frac{\sigma\left(a_{1}+a_{2}\right)}{b_{1} b_{2}}},
$$

provided $\mu_{*} \in[0,1]$. The latter condition holds if $a_{1}, a_{1}>0, \sigma b_{1} \leq b_{2}$, and $\sigma b_{2} \leq b_{1}$. In particular, it holds if $\sigma \leq 0$; that is, the risky assets are negatively correlated.

Substituting $\mu=\mu_{*}$ to (4) and (5) we obtain

$$
\begin{gathered}
\widetilde{r} k^{*}(s)=\frac{\frac{a_{1}^{2}}{b_{1}^{2}}+\frac{a_{2}^{2}}{b_{2}^{2}}-\frac{\sigma a_{1} a_{2}}{b_{1} b_{2}}}{a_{1}^{2}\left(\frac{1}{b_{1}}-\frac{\sigma}{b_{2}}\right)^{2}+a_{2}^{2}\left(\frac{1}{b_{2}}-\frac{\sigma}{b_{1}}\right)^{2}+2 \sigma a_{1} a_{2}\left(\frac{1}{b_{1}}-\frac{\sigma}{b_{2}}\right)\left(\frac{1}{b_{2}}-\frac{\sigma}{b_{1}}\right)}, \\
k^{*}(s)=\frac{1}{\widetilde{r}} \cdot \frac{\frac{a_{1}^{2}}{b_{1}^{2}}+\frac{a_{2}^{2}}{b_{2}^{2}}-\frac{\sigma a_{1} a_{2}}{b_{1} b_{2}}}{a_{1}^{2}\left(\frac{1}{b_{1}}-\frac{\sigma}{b_{2}}\right)^{2}+a_{2}^{2}\left(\frac{1}{b_{2}}-\frac{\sigma}{b_{1}}\right)^{2}+2 \sigma a_{1} a_{2}\left(\frac{1}{b_{1}}-\frac{\sigma}{b_{2}}\right)\left(\frac{1}{b_{2}}-\frac{\sigma}{b_{1}}\right)},
\end{gathered}
$$


where $\widetilde{r}$ is the maximal root of the equation

$$
\lambda h(r)=c r+\frac{1}{2} \cdot \frac{\left(\frac{a_{1}^{2}}{b_{1}^{2}}+\frac{a_{2}^{2}}{b_{2}^{2}}-\frac{\sigma a_{1} a_{2}}{b_{1} b_{2}}\right)^{2}}{a_{1}^{2}\left(\frac{1}{b_{1}}-\frac{\sigma}{b_{2}}\right)^{2}+a_{2}^{2}\left(\frac{1}{b_{2}}-\frac{\sigma}{b_{1}}\right)^{2}+2 \sigma a_{1} a_{2}\left(\frac{1}{b_{1}}-\frac{\sigma}{b_{2}}\right)\left(\frac{1}{b_{2}}-\frac{\sigma}{b_{1}}\right)} .
$$

In the case of uncorrelated assets, that is, in the case of $\sigma=0$, equation (8) becomes

$$
\lambda h(r)=c r+\frac{a_{1}^{2}}{2 b_{1}^{2}}+\frac{a_{2}^{2}}{2 b_{2}^{2}} .
$$

If $\mu_{*}>1$, then the insurer should invest in the first asset, while if $\mu_{*}<0$, then the insurer should invest in the second asset.

The root of equation (2) is not less than the root corresponding to the case of a single asset, since the second term of the right hand side of equation (5) for $\mu_{*} \in(0,1)$ is greater than that for $\mu=1$ or for $\mu=0$. The latter two cases correspond to the investment in a single asset only.

Remark 2.1. A similar reasoning for the case of $m$ different risky assets (modelled by jointly independent geometric Brownian motions) leads to the upper bound $\Psi(x) \leq e^{-\widetilde{r} x}$ for the ruin probability, where $\widetilde{r}$ is the maximal root of the equation

$$
\lambda h(r)=c r+\frac{a_{1}^{2}}{2 b_{1}^{2}}+\frac{a_{2}^{2}}{2 b_{2}^{2}}+\cdots+\frac{a_{m}^{2}}{2 b_{m}^{2}} .
$$

If the number of risky assets available at the market increases, then the survival probability increases, too.

The strategies $k_{1}^{*}=\mu_{*} k^{*}$ and $k_{2}^{*}=\left(1-\mu_{*}\right) k^{*}$ found above are suitable for the case where a free credit is available in the money market. This strategy suggests investing a constant amount in each asset. It is clear that the investment in the asset $i$ does not make sense if $a_{i}<0$, since this asset is nonprofitable. Thus an optimal strategy would suggest that the insurer sell the asset $i$, which is impossible.

Since the increments of $Y\left(t, x, k_{1}^{*}, k_{2}^{*}\right)$ are stationary and independent, we have, for $0 \leq t \leq T$, that

$$
\begin{aligned}
\mathrm{E}\left[M\left(T, x, k_{1}^{*}, k_{2}^{*}, \widetilde{r}\right) \mid F_{t}\right] & =\mathrm{E}\left[e^{-\widetilde{r} Y\left(T, x, k_{1}^{*}, k_{2}^{*}\right)} \mid F_{t}\right] \\
& =e^{-\widetilde{r} Y\left(t, x, k_{1}^{*}, k_{2}^{*}\right)} \mathrm{E}\left[e^{-\widetilde{r}\left(Y\left(T, x, k_{1}^{*}, k_{2}^{*}\right)-Y\left(t, x, k_{1}^{*}, k_{2}^{*}\right)\right)} \mid F_{t}\right] \\
& =e^{-\widetilde{r} Y\left(t, x, k_{1}^{*}, k_{2}^{*}\right)} \mathrm{E}\left[e^{-\widetilde{r}\left(Y\left(T-t, x, k_{1}^{*}, k_{2}^{*}\right)-Y\left(0, x, k_{1}^{*}, k_{2}^{*}\right)\right)}\right] \\
& =e^{-\widetilde{r} Y\left(t, x, k_{1}^{*}, k_{2}^{*}\right)} \mathrm{E}\left[e^{-\widetilde{r} Y\left(T-t, 0, k_{1}^{*}, k_{2}^{*}\right)}\right]=e^{-\widetilde{r} Y\left(t, x, k_{1}^{*}, k_{2}^{*}\right)} \\
& =M\left(t, x, k_{1}^{*}, k_{2}^{*}, \widetilde{r}\right) .
\end{aligned}
$$

Therefore $M\left(t, x, k_{1}^{*}, k_{2}^{*}, \widetilde{r}\right)$ is an $F_{t}$-martingale.

Theorem 2.2. Let $a_{i} \neq 0$ and $b_{i} \neq 0, i=1,2$. If the investment strategies $k_{1}^{*}(t)=$ $\mu_{*} k^{*}(t)$ and $k_{2}^{*}(t)=\left(1-\mu_{*}\right) k^{*}(t)$, where $k^{*}(t)$ is defined by equality (7), are constant, then the ruin probability is bounded from above, namely

$$
\Psi\left(x, k_{1}^{*}, k_{2}^{*}\right) \leq e^{-\widetilde{r} x}
$$

for all $x \geq 0$.

Proof. We follow the lines of the proof in the paper [1. Consider the processes $M$ and $Y$ at the ruin moment; that is, consider

$$
\bar{M}\left(t, x, k_{1}, k_{2}, r\right)=M\left(t \wedge \tau\left(x, k_{1}, k_{2}\right), x, k_{1}, k_{2}, r\right)
$$


and

$$
\bar{Y}\left(t, x, k_{1}, k_{2}\right)=Y\left(t \wedge \tau\left(x, k_{1}, k_{2}\right), x, k_{1}, k_{2}\right),
$$

where $t \wedge \tau\left(x, k_{1}, k_{2}\right)=\min \left(t, \tau\left(x, k_{1}, k_{2}\right)\right)$.

Since $M\left(t, x, k_{1}^{*}, k_{2}^{*}, \widetilde{r}\right)$ is an $F_{t}$-martingale, the stopped process $\bar{M}\left(t, x, k_{1}^{*}, k_{2}^{*}, \widetilde{r}\right)$ is also an $F_{t}$-martingale. The process $\bar{M}$ is nonnegative for $t \geq 0$, thus

$$
\begin{aligned}
e^{-\widetilde{r} x}= & \bar{M}\left(0, x, k_{1}^{*}, k_{2}^{*}, \widetilde{r}\right)=\mathrm{E}\left[\bar{M}\left(t, x, k_{1}^{*}, k_{2}^{*}, \widetilde{r}\right)\right] \\
= & \mathrm{E}\left[\bar{M}\left(\tau\left(x, k_{1}^{*}, k_{2}^{*}\right), x, k_{1}^{*}, k_{2}^{*}, \widetilde{r}\right) \chi_{\left\{\tau\left(x, k_{1}^{*}, k_{2}^{*}\right)<t\right\}}\right] \\
& +\mathrm{E}\left[\bar{M}\left(t, x, k_{1}^{*}, k_{2}^{*}, \widetilde{r}\right) \chi_{\left.\left\{\tau\left(x, k_{1}^{*}, k_{2}^{*}\right) \geq t\right\}\right]}\right. \\
\geq & \mathrm{E}\left[\bar{M}\left(\tau\left(x, k_{1}^{*}, k_{2}^{*}\right), x, k_{1}^{*}, k_{2}^{*}, \widetilde{r}\right) \chi_{\left\{\tau\left(x, k_{1}^{*}, k_{2}^{*}\right)<t\right\}}\right],
\end{aligned}
$$

where the symbol $\chi_{A}$ denotes the indicator of a set $A$.

By the monotone convergence theorem,

$$
\begin{aligned}
\lim _{t \rightarrow \infty} \mathrm{E} & {\left[\bar{M}\left(\tau\left(x, k_{1}^{*}, k_{2}^{*}\right), x, k_{1}^{*}, k_{2}^{*}, \widetilde{r}\right) \chi_{\left\{\tau\left(x, k_{1}^{*}, k_{2}^{*}\right)<t\right\}}\right] } \\
= & \mathrm{E}\left[\bar{M}\left(\tau\left(x, k_{1}^{*}, k_{2}^{*}\right), x, k_{1}^{*}, k_{2}^{*}, \widetilde{r}\right) \chi_{\left\{\tau\left(x, k_{1}^{*}, k_{2}^{*}\right)<\infty\right\}}\right] .
\end{aligned}
$$

Hence

$$
e^{-\widetilde{r} x} \geq \mathrm{E}\left[\bar{M}\left(\tau\left(x, k_{1}^{*}, k_{2}^{*}\right), x, k_{1}^{*}, k_{2}^{*}, \widetilde{r}\right) \mid \tau\left(x, k_{1}^{*}, k_{2}^{*}\right)<\infty\right] \cdot \mathrm{P}\left\{\tau\left(x, k_{1}^{*}, k_{2}^{*}\right)<\infty\right\} .
$$

Now we obtain an upper bound for the ruin probability:

$$
\begin{aligned}
\Psi\left(x, k_{1}^{*}, k_{2}^{*}\right) & =\mathrm{P}\left\{\tau\left(x, k_{1}^{*}, k_{2}^{*}\right)<\infty\right\} \\
& \leq \frac{e^{-\widetilde{r} x}}{\mathrm{E}\left[\bar{M}\left(\tau\left(x, k_{1}^{*}, k_{2}^{*}\right), x, k_{1}^{*}, k_{2}^{*}, \widetilde{r}\right) \mid \tau\left(x, k_{1}^{*}, k_{2}^{*}\right)<\infty\right]} \leq e^{-\widetilde{r} x},
\end{aligned}
$$

since the random variable $\bar{M}\left(\tau\left(x, k_{1}^{*}, k_{2}^{*}\right), x, k_{1}^{*}, k_{2}^{*}, \widetilde{r}\right)$ is not less than 1 almost surely on the set $\left\{\tau\left(x, k_{1}^{*}, k_{2}^{*}\right)<\infty\right\}$.

Theorem 2.1 follows from Theorem 2.2

2.3. A bound for the ruin probability in the case where a free credit is not available. Since a free credit is not always available in a money market, an interesting problem is to evaluate the probability $\varphi\left(x, k_{1}, k_{2}\right)$ that an insurer can follow the above strategy without free credit. The optimal strategy suggests that the insurer will invest a constant amount $k^{*}$ in a risky asset. Thus, this amount of money should be available at any time; that is, the capital should be at least $k^{*}$ at any time. Therefore,

$$
\begin{aligned}
\varphi\left(x, k_{1}, k_{2}\right) & =\mathrm{P}\left\{Y\left(t, x, k_{1}, k_{2}\right) \geq k^{*} \text { for all } t \geq 0\right\} \\
& =1-\mathrm{P}\left\{Y\left(t, x, k_{1}, k_{2}\right)<k^{*} \text { for some } t \geq 0\right\} \geq 1-e^{-\widetilde{r}\left(x-k^{*}\right)} .
\end{aligned}
$$

This implies that the ruin probability $\Psi_{1}\left(x, k_{1}^{*}, k_{2}^{*}\right)$ for an insurer can be estimated as follows:

$$
\begin{aligned}
\Psi_{1}\left(x, k_{1}^{*}, k_{2}^{*}\right) & =\mathrm{P}\left\{Y\left(t, x, k_{1}, k_{2}\right)<0 \text { for some } t \geq 0\right\} \\
& \leq \mathrm{P}\left\{Y\left(t, x, k_{1}, k_{2}\right)<k^{*} \text { for some } t \geq 0\right\} \leq e^{-\widetilde{r}\left(x-k^{*}\right)}
\end{aligned}
$$

if a free credit is not available in the money market. 
2.4. The investment strategy for the case where a free credit is not available in the money market. We assume that $w_{1}(t)$ and $w_{2}(t)$ are independent. If a free credit is not available in the money market, the strategies $k_{1}^{*}$ and $k_{2}^{*}$ become useless in the case of $Y\left(t, x, k_{1}, k_{2}\right)<k_{1}^{*}+k_{2}^{*}$, that is, if the capital at the moment $t$ is less than the total amount needed to invest in assets.

Assume that an insurer can invest an amount $k_{0}$. For example, this is the case if

$$
Y\left(t, x, k_{1}, k_{2}\right)=k_{0}
$$

This means that either the insurer possesses the capital $k_{0}$ at the moment $t$ or that the strategy is to invest the amount $k_{0}$.

Put $k_{1}=k_{0} \mu$ and $k_{2}=k_{0}(1-\mu)$, where $\mu$ is the fraction of the money $k_{0}$ invested in the first risky asset.

According to the reasoning above one should maximize the following expression:

$$
r k_{0}\left(\mu a_{1}+(1-\mu) a_{2}\right)-\frac{1}{2} r^{2} k_{0}^{2}\left(\mu^{2} b_{1}^{2}+(1-\mu)^{2} b_{2}^{2}\right) .
$$

This expression attains its maximum at the point

$$
r k_{0}=\frac{\mu a_{1}+(1-\mu) a_{2}}{\mu^{2} b_{1}^{2}+(1-\mu)^{2} b_{2}^{2}} .
$$

Substituting this value of $r k_{0}$ in $(2)$ we get

$$
\lambda h(r)=c r+\frac{\left(\mu a_{1}+(1-\mu) a_{2}\right)^{2}}{2\left(\mu^{2} b_{1}^{2}+(1-\mu)^{2} b_{2}^{2}\right)} .
$$

The second term on the right hand side attains its maximum if

$$
\mu=\mu_{*}=\frac{\frac{a_{1}}{b_{1}^{2}}}{\frac{a_{1}}{b_{1}^{2}}+\frac{a_{2}}{b_{2}^{2}}} .
$$

Equation (41) with $\mu=\mu_{*}$ takes the form

$$
\lambda h(r)=c r+\frac{1}{2}\left(\frac{a_{1}^{2}}{b_{1}^{2}}+\frac{a_{2}^{2}}{b_{2}^{2}}\right) .
$$

Substituting $\mu=\mu_{*}$ in (3) we obtain $r k_{0}=a_{1} / b_{1}^{2}+a_{2} / b_{2}^{2}$, that is,

$$
k_{0}^{*}=\frac{1}{\widetilde{r}}\left(\frac{a_{1}}{b_{1}^{2}}+\frac{a_{2}}{b_{2}^{2}}\right) \text {, }
$$

where $\widetilde{r}$ is the maximal root of the equation

$$
\lambda h(r)=c r+\frac{1}{2}\left(\frac{a_{1}^{2}}{b_{1}^{2}}+\frac{a_{2}^{2}}{b_{2}^{2}}\right) .
$$

We obtained the same equation for $\widetilde{r}$ and $k_{0}^{*}=k_{1}^{*}+k_{2}^{*}$ where $k_{1}^{*}$ and $k_{2}^{*}$ are evaluated above for the case where a free credit is available.

Since one can find a maximum with respect to $\mu$ and then with respect to $k_{0}$, the latter result means that the insurer should distribute the amounts for investments according to the proportion $\mu:(1-\mu)$ even if $Y\left(t, x, k_{1}, k_{2}\right)$ is less than the optimal amount $k_{0}^{*}$. 


\section{A BOUND FOR THE RUIN PROBABILITY AND THE OPTIMAL INVESTMENT STRATEGY} FOR THE SEMIMARTINGALE MODEL WITH SEVERAL RISKY ASSETS

3.1. The model. Now we consider another model for the risk process of an insurer. Let the capital of the insurer at the moment $t \geq 0$ be described by the equation

$$
Y\left(t, x, \phi_{s}^{1}, \phi_{s}^{2}\right)=R_{1}(t)+R_{2}(t),
$$

where

$$
R_{1}(t)=x+c t-\sum_{i=1}^{N(t)} X_{i}, \quad R_{2}(t)=\int_{0}^{t} \frac{\phi_{s}^{1}}{S_{s}^{1}} d S_{s}^{1}+\int_{0}^{t} \frac{\phi_{s}^{2}}{S_{s}^{2}} d S_{s}^{2} .
$$

The classical component $R_{1}(t)$ is assumed to be the same as that used in the model described by equation (11). The second component $R_{2}(t)$ reflects the assumption that the insurer invests in two different risky assets whose price processes $S_{t}^{i}, i=1,2$, are almost surely positive and admit the following stochastic differentials:

$$
d S_{t}^{i}=S_{t}^{i}\left(d M_{t}^{i}+d A_{t}^{i}\right), \quad t \geq 0, i=1,2,
$$

where the $M_{t}^{i}$ are square-integrable continuous martingales whose square characteristics $\left\langle M^{i}\right\rangle_{t}$ are absolutely continuous with respect to Lebesgue measure; that is,

$$
\left\langle M^{i}\right\rangle_{t}=\int_{0}^{t} \beta_{s}^{i} d s \text { and } \int_{0}^{t}\left|\beta_{s}^{i}\right| d s<\infty
$$

almost surely for all $t>0$, and where the variations of the processes $A_{t}^{i}$ are integrable, that is,

$$
A_{t}^{i}=\int_{0}^{t} \alpha_{s}^{i} d s \quad \text { and } \quad \int_{0}^{t}\left|\alpha_{s}^{i}\right| d s<\infty
$$

almost surely for all $t>0$.

In contrast to Section 2, the processes $S_{t}^{1}$ and $S_{t}^{2}$ are assumed to be independent, hence $\left\langle M^{1}, M^{2}\right\rangle_{t} \equiv 0$.

The random variable $\phi_{t}^{i}$ is equal to the amount of money invested by the insurer in the asset $i$ at the moment $t, t \geq 0, i=1,2$.

We also assume that the processes $\phi_{t}^{i}$ are $F_{t}^{i}$-predictable where

$$
F_{t}^{i}=\sigma\left\{R_{1}(s), S_{s}^{i}, 0 \leq s \leq t\right\}=\sigma\left\{R_{1}(s), S_{s}^{i}, M_{s}^{i}, A_{s}^{i}, 0 \leq s \leq t\right\}, \quad i=1,2,
$$

and such that

$$
\mathrm{E} \int_{0}^{t}\left(\phi_{s}^{i}\right)^{2} \beta_{s}^{i} d s<\infty, \quad \int_{0}^{t}\left|\phi_{s}^{i} \alpha_{s}^{i}\right| d s<\infty, \quad i=1,2 .
$$

Then

$$
\int_{0}^{t} \frac{\phi_{s}^{i}}{S_{s}^{i}} d S_{s}^{i}=\int_{0}^{t} \phi_{s}^{i} d M_{s}^{i}+\int_{0}^{t} \phi_{s}^{i} d A_{s}^{i}
$$

where $\int_{0}^{t} \phi_{s}^{i} d M_{s}^{i}$ is an integrable martingale, since it is the Itô integral with respect to a square-integrable martingale. Here $\int_{0}^{t} \phi_{s}^{i} d A_{s}^{i}$ is the Lebesgue-Stieltjes integral, $i=1,2$.

Equation (10) is rewritten in the form

$$
Y\left(t, x, \phi^{1}, \phi^{2}\right)=x+c t-\sum_{i=1}^{N(t)} X_{i}+\int_{0}^{t} \phi_{s}^{1} d M_{s}^{1}+\int_{0}^{t} \phi_{s}^{1} d A_{s}^{1}+\int_{0}^{t} \phi_{s}^{2} d M_{s}^{2}+\int_{0}^{t} \phi_{s}^{2} d A_{s}^{2} .
$$

We also assume that $M^{1}, M^{2}, A^{1}, A^{2}, \phi^{1}$, and $\phi^{2}$ are jointly independent of the sequence $X_{i}, i \geq 1$, and process $N$. 
3.2. The optimal strategy and a bound for the ruin probability. Consider a positive stochastic process

$$
Z\left(t, x, \phi^{1}, \phi^{2}, r\right)=\exp \left\{-r Y\left(t, x, \phi^{1}, \phi^{2}\right)\right\}, \quad r>0 .
$$

We look for conditions that $Z\left(\cdot, x, \phi^{1}, \phi^{2}, r\right)$ is an $F_{t}$-supermartingale, where

$$
F_{t}=\sigma\left(F_{t}^{1}, F_{t}^{2}\right)
$$

As in Section 2, put

$$
\begin{gathered}
h(r)=\mathrm{E} \exp \{r X\}-1, \\
f\left(t, \phi^{1}, \phi^{2}, r\right)=\lambda h(r)-c r+\frac{r^{2}}{2}\left(\left(\phi_{t}^{1}\right)^{2} \beta_{t}^{1}+\left(\phi_{t}^{2}\right)^{2} \beta_{t}^{2}\right)-r\left(\phi_{t}^{1} \alpha_{t}^{1}+\phi_{t}^{2} \alpha_{t}^{2}\right) .
\end{gathered}
$$

Theorem 3.1. Suppose that, for a number $r>0$ and a strategy $\left\{\phi_{t}^{1}, \phi_{t}^{2} \geq 0\right\}$,

(1) $\mathrm{E} \exp \left\{r X_{1}\right\}<\infty$, and

(2) $f\left(t, \phi^{1}, \phi^{2}, r\right) \leq 0, t \geq 0$, almost surely.

Then the process $\left\{Z\left(t, x, \phi^{1}, \phi^{2}, r\right), t \geq 0\right\}$ is an $F_{t}$-supermartingale.

Proof. The inequality

$$
\mathrm{E} \exp \left(-r \int_{0}^{t} \phi_{s}^{i} d M_{s}^{i}-\frac{r^{2}}{2} \int_{0}^{t}\left(\phi_{s}^{i}\right)^{2} \beta_{s}^{i} d s\right) \leq 1, \quad i=1,2, t \geq 0
$$

holds according to Theorem 5.2 of [6]. Assumption (2) and the independence of the families $\left\{M^{1}, M^{2}, A^{1}, A^{2}, \phi^{1}, \phi^{2}\right\}$ and $\left\{X_{i}, i \geq 1, N\right\}$ imply that

$$
\begin{aligned}
\mathrm{E} Z( & \left.t, 0, \phi^{1}, \phi^{2}, r\right) \\
= & e^{-r c t} \mathrm{E} \exp \left\{r \sum_{i=1}^{N(t)} X_{i}\right\} \\
& \times \mathrm{E} \exp \left\{-r \int_{0}^{t} \phi_{s}^{1} d M_{s}^{1}-r \int_{0}^{t} \phi_{s}^{1} \alpha_{s}^{1} d s-r \int_{0}^{t} \phi_{s}^{2} d M_{s}^{2}-\int_{0}^{t} \phi_{s}^{2} \alpha_{s}^{2} d s\right\} \\
= & \exp \{-r c t+h(r) \lambda t\} \\
& \times \operatorname{E} \exp \left\{-r \int_{0}^{t} \phi_{s}^{1} d M_{s}^{1}-r \int_{0}^{t} \phi_{s}^{1} \alpha_{s}^{1} d s-r \int_{0}^{t} \phi_{s}^{2} d M_{s}^{2}-r \int_{0}^{t} \phi_{s}^{2} \alpha_{s}^{2} d s\right\} \\
= & \exp \{-r c t+h(r) \lambda t\} \\
& \times\left(\operatorname{E} \exp \left\{-r \sum_{i=1}^{2} \int_{0}^{t} \phi_{s}^{i} d M_{s}^{i}-\frac{r^{2}}{2} \sum_{i=1}^{2} \int_{0}^{t}\left(\phi_{s}^{i}\right)^{2} \beta_{s}^{i} d s\right\}\right. \\
& \left.\times \exp \left\{\frac{r^{2}}{2} \sum_{i=1}^{2} \int_{0}^{t}\left(\phi_{s}^{i}\right)^{2} \beta_{s}^{i} d s-r \sum_{i=1}^{2} \int_{0}^{t} \phi_{s}^{i} \alpha_{s}^{i} d s\right\}\right) \leq 1
\end{aligned}
$$

for all $t>0$, since $M^{1}$ and $M^{2}$ are orthogonal. This means that $Z\left(t, x, \phi^{1}, \phi^{2}, r\right)$ is an integrable process.

Since $\sum_{i=N\left(t_{1}\right)+1}^{N\left(t_{2}\right)} X_{i}$ does not depend on $F_{t_{1}}$ and on the family

$$
\left\{M^{1}, M^{2}, A^{1}, A^{2}, \phi^{1}, \phi^{2}\right\}
$$


for $0 \leq t_{1} \leq t_{2}$, we have, by assumption (2), that

$$
\begin{aligned}
& \mathrm{E} Z\left(t_{2}, x, \phi^{1}, \phi^{2}, r \mid F_{t_{1}}\right) \\
& =Z\left(t_{1}, x, \phi^{1}, \phi^{2}, r\right) \exp \left\{-r c\left(t_{2}-t_{1}\right)\right\} \\
& \times \mathrm{E}\left[\prod _ { i = 1 } ^ { 2 } \left(\exp \left\{-r \int_{t_{1}}^{t_{2}} \phi_{s}^{i} d M_{s}^{i}-\frac{r^{2}}{2} \int_{t_{1}}^{t_{2}}\left(\phi_{s}^{i}\right)^{2} \beta_{s}^{i} d s\right\}\right.\right. \\
& \left.\left.\quad \times \exp \left\{\frac{r^{2}}{2} \int_{t_{1}}^{t_{2}}\left(\phi_{s}^{i}\right)^{2} \beta_{s}^{i} d s-r \int_{t_{1}}^{t_{2}}\left(\phi_{s}^{i}\right)^{2} \beta_{s}^{i} d s\right\}\right) \mid F_{t_{1}}\right] \\
& =Z\left(t_{1}, x, \phi^{1}, \phi^{2}, r\right) \mathrm{E}\left[\prod _ { i = 1 } ^ { 2 } \left(\exp \left\{-r \int_{t_{1}}^{t_{2}} \phi_{s}^{i} d M_{s}^{i}-\frac{r^{2}}{2} \int_{t_{1}}^{t_{2}}\left(\phi_{s}^{i}\right)^{2} \beta_{s}^{i} d s\right\}\right.\right. \\
& \leq Z\left(t_{1}, x, \phi^{1}, \phi^{2}, r\right) \cdot \mathrm{E}\left[\prod_{i=1}^{2}\left(\exp \left\{-r \int_{t_{1}}^{t_{2}} f\left(s, \phi_{s}^{1}, \phi^{2}, r\right) d s\right\}\right) \mid F_{t_{1}}\right] \\
& \left.\left.\left.\leq Z\left(t_{1}, x, \phi_{s}^{1}, \phi^{2}, r\right) . \frac{r^{2}}{2} \int_{t_{1}}^{t_{2}}\left(\phi_{s}^{i}\right)^{2} \beta_{s}^{i} d s\right\}\right) \mid F_{t_{1}}\right]
\end{aligned}
$$

It is necessary to choose the parameter $r$ as large as possible. In particular, it should be greater than the positive root of the equation $\lambda h(r)=c r$, since the bound for the ruin probability corresponding to this root becomes sharp for the strategy $\left\{\phi_{s}^{1} \equiv 0, \phi_{s}^{2} \equiv 0\right.$, $s \geq 0\}$, that is, for the case where the insurer does not invest at all.

Now we find strategies satisfying assumption (2) of Theorem 3.1 and evaluate the maximal value of the parameter $r>0$.

Theorem 3.2. Let the functions $\alpha^{i}$ and $\beta^{i}, i=1,2$, be such that

(1) $\beta_{s}^{i}>0$ for $s \geq 0$ and $i=1,2$;

(2) there exist constants $c_{1}, c_{2}>0$ for which

$$
\underset{s \geq 0}{\operatorname{ess} \inf _{s}} \frac{\left(\alpha_{s}^{i}\right)^{2}}{2 \beta_{s}^{i}} \geq c_{i} \quad \text { almost surely, } \quad i=1,2,
$$

where

$$
\underset{s \geq 0}{\operatorname{ess} \inf }=\sup _{A: \lambda(A)=0} \inf _{s \in \mathbb{R} \backslash A}
$$

and $\lambda$ is Lebesgue measure on $\mathbb{R}_{+}$.

Then there exists a nonzero predictable strategy $\left\{\phi_{s}^{1}, \phi_{s}^{2}, s \geq 0\right\}$ satisfying assumption (2) of Theorem 3.1. Moreover, one can choose $r=\widetilde{r}$, where $\widetilde{r}$ is a unique positive root of the equation $\lambda h(r)=c r+c_{1}+c_{2}$.

Proof. To satisfy assumption (2) of Theorem 3.1, that is, to satisfy the inequality

$$
f\left(s, \phi^{1}, \phi^{2}, \widetilde{r}\right) \leq 0,
$$

note that the latter inequality is equivalent to

$$
\left(\frac{\widetilde{r}^{2}}{2}\left(\phi_{s}^{1}\right)^{2} \beta_{s}^{1}-\widetilde{r} \phi_{s}^{1} \alpha_{s}^{1}+c_{1}\right)+\left(\frac{\widetilde{r}^{2}}{2}\left(\phi_{s}^{2}\right)^{2} \beta_{s}^{2}-\widetilde{r} \phi_{s}^{2} \alpha_{s}^{2}+c_{2}\right) \leq 0 .
$$

The discriminants of the left hand side of inequality (13) are equal to

$$
D^{i}(s, \omega)=\widetilde{r}^{2} \alpha_{s}^{i}-2 \widetilde{r}^{2} c_{i} \beta_{s}^{i}
$$


for $i=1,2$. Since

$$
\underset{s \geq 0}{\operatorname{ess} \inf _{s}} \frac{\left(\alpha_{s}^{i}\right)^{2}}{2 \beta_{s}^{i}} \geq c_{i}>0
$$

we get $D^{i}(s, \omega) \geq 0(\bmod \lambda \times \mathrm{P})$ for $\left(\alpha_{s}^{i}\right)^{2} /\left(2 \beta_{s}^{i}\right) \geq c_{i}$. Then we put

$$
\phi_{s}^{i} \in\left[\frac{\alpha_{s}^{i}-\sqrt{\left(\alpha_{s}^{i}\right)^{2}-2 c_{i} \beta_{s}^{i}}}{\widetilde{r} \beta_{s}^{i}}, \frac{\alpha_{s}^{i}+\sqrt{\left(\alpha_{s}^{i}\right)^{2}-2 c_{i} \beta_{s}^{i}}}{\widetilde{r} \beta_{s}^{i}}\right], \quad i=1,2,
$$

for those $s$ where $D^{i}(s, \omega) \geq 0$, and $\phi_{s}^{i}=0$ for other points $s$. Assumption (2) of Theorem 3.1 is satisfied.

To satisfy conditions (12) we put

$$
\phi_{s}^{i}=\frac{\alpha_{s}^{i}-\sqrt{\left(\alpha_{s}^{i}\right)^{2}-2 c_{i} \beta_{s}^{i}}}{\widetilde{r} \beta_{s}^{i}}=\frac{2 c_{i}}{\widetilde{r}\left(\alpha_{s}^{i}+\sqrt{\left(\alpha_{s}^{i}\right)^{2}-2 c_{i} \beta_{s}^{i}}\right)}, \quad i=1,2 .
$$

Then $\alpha_{s}^{i} \phi_{s}^{i} \widetilde{r} \leq 2 \widetilde{r} c_{i}$, whence

$$
\frac{\widetilde{r}^{2}}{2}\left(\phi_{s}^{i}\right)^{2} \beta_{s}^{i} \leq \widetilde{r} \phi_{s}^{i} \alpha_{s}^{i}-c_{i} \leq c_{i}
$$

Thus conditions (12) hold for all $t>0$.

Remark 3.1. If $\beta_{s}^{i}=0, i=1,2$, then the inequality $f\left(s, \phi^{1}, \phi^{2}, \widetilde{r}\right) \leq 0$ can be rewritten in the form

$$
-\widetilde{r}\left(\phi_{s}^{1} \alpha_{s}^{1}+\phi_{s}^{2} \alpha_{s}^{2}\right)+c_{1}+c_{2} \leq 0 .
$$

Suppose the prices of the assets become nonrandom at a certain moment; then the insurer should invest as much money as possible in the assets if $\alpha_{s}^{i}>0$, and avoid investing if $\alpha_{s}^{i}<0$.

Remark 3.2. Since $h(0)=0$ and the function $h(r)$ is concave and increasing, the equation

$$
\lambda h(r)-c r=c_{1}+c_{2}
$$

has a unique positive root $\widetilde{r}=\widetilde{r}\left(c_{1}+c_{2}\right)$ if $c_{1}+c_{2}>0$. The root increases with $c_{1}+c_{2}$. If $c_{i}>0, i=1,2$, then $\widetilde{r}\left(c_{1}+c_{2}\right)>\widetilde{r}\left(c_{i}\right)$. This means that an investment in two different assets brings an insurer an extra profit as compared to the case of a single asset considered in the paper [2].

Let $\tau\left(x, \phi^{1}, \phi^{2}\right)=\inf \left\{t>0: Y\left(t, x, \phi^{1}, \phi^{2}\right)<0\right\}$ be the ruin moment of an insurer, and let

$$
\Psi\left(x, \phi_{s}^{1}, \phi_{s}^{2}\right)=\mathrm{P}\left\{Y\left(t, x, \phi_{s}^{1}, \phi_{s}^{2}\right)<0 \text { for some } t \geq 0\right\}
$$

be the ruin probability depending on the initial capital $x$ and on the investment strategy chosen by the insurer.

Theorem 3.3. Let assumptions of Theorem 3.2 hold and let the strategy $\left\{\phi_{s}^{1}, \phi_{s}^{2}, s \geq 0\right\}$ be chosen according to equality (15). Then the ruin probability admits the upper bound

$$
\Psi\left(x, \phi_{s}^{1}, \phi_{s}^{2}\right) \leq e^{-\tilde{r} x},
$$

where $\widetilde{r}=\widetilde{r}\left(c_{1}+c_{2}\right)$ is a unique positive root of the equation $\lambda h(r)=c r+c_{1}+c_{2}$.

The proof of Theorem 3.3 is similar to that of Theorem 3 in the paper [2] and to the proof of Theorem 2.2 given in Section 2. 
M. V. BRATYK

Remark 3.3. The following results can be proved similarly to the case of $n=2$ different risky assets. We assume that the price processes are described in the same way as in Section 3.1 provided they are jointly independent semimartingales possessing absolutely continuous characteristics with respect to Lebesgue measure.

Theorem 3.4. Let the functions $\alpha^{i}$ and $\beta^{i}, i=1, \ldots, n$, be such that

1) $\beta_{s}^{i}>0$ for $s \geq 0$ and $i=1, \ldots, n$;

2) there exist constants $c_{i}>0$ such that

$$
\underset{s \geq 0}{\operatorname{ess} \inf } \frac{\left(\alpha_{s}^{i}\right)^{2}}{2 \beta_{s}^{i}} \geq c_{i} \quad \text { almost surely, } \quad i=1, \ldots, n .
$$

Then there exists a nonzero predictable strategy $\left\{\phi_{s}^{1}, \ldots, \phi_{s}^{n}, s \geq 0\right\}$ such that

$$
f\left(t, \phi^{1}, \ldots, \phi^{n}, r\right) \leq 0, \quad t \geq 0,
$$

almost surely, where

$$
f\left(t, \phi^{1}, \ldots, \phi^{n}, r\right)=\lambda h(r)-c r+\frac{r^{2}}{2}\left(\sum_{i=1}^{n}\left(\phi_{t}^{i}\right)^{2} \beta_{t}^{i}\right)-r\left(\sum_{i=1}^{n} \phi_{t}^{i} \alpha_{t}^{i}\right) .
$$

Moreover one can choose $r=\widetilde{r}$ where $\widetilde{r}$ is a unique positive root of the equation

$$
\lambda h(r)=c r+\sum_{i=1}^{n} c_{i} .
$$

As in Section 3.1, $\phi_{t}^{i}$ means the amount of money invested by the insurer in the asset $i$ at the moment $t, t \geq 0, i=1, \ldots, n$.

Theorem 3.5. Let the assumptions of Theorem 3.4 hold. Let the strategy

$$
\left\{\phi_{s}^{1}, \ldots, \phi_{s}^{n}, s \geq 0\right\}
$$

be such that

$$
\phi_{s}^{i}=\frac{\alpha_{s}^{i}-\sqrt{\left(\alpha_{s}^{i}\right)^{2}-2 c_{i} \beta_{s}^{i}}}{\widetilde{r} \beta_{s}^{i}}, \quad i=1, \ldots, n .
$$

Then

$$
\Psi\left(x, \phi_{s}^{1}, \ldots, \phi_{s}^{n}\right) \leq e^{-\widetilde{r} x},
$$

where $\widetilde{r}=\widetilde{r}\left(c_{1}+c_{2}+\cdots+c_{n}\right)$ is a unique positive root of the equation

$$
\lambda h(r)=c r+\sum_{i=1}^{n} c_{i} .
$$

\section{Concluding Remarks}

We have obtained an upper bound $\Psi(x) \leq e^{-r x}$ for the ruin probability for the case where the insurer invests in several risky assets modelled by either geometric Brownian motions or semimartingales. We improve the coefficient $r$ in this bound as compared to the cases where the insurer does not invest at all or where there is only a single risky asset. In particular, if the price processes are modelled by geometric Brownian motions, the above bound corresponds to the investment strategy that suggests that the insurer will invest constant amounts of money in every risky asset, independently of the current capital. For this case, we found an upper bound under the assumption that a free credit is not available in the money market.

The results of Section 2 are generalized to the case where there are several risky assets whose price processes are described by jointly independent geometric Brownian motions. 


\section{BIBLIOGRAPHY}

1. J. Gaier, P. Grandits, and W. Schachermayer, Asymptotic ruin probabilities and optimal investment, Ann. Appl. Probab. 13 (2003), no. 3, 1054-1076. MR.1994044(2004k:91124)

2. Yu. S. Mishura, An estimate for the ruin probability for models with long-term dependence, Teor. Imovir. Mat. Stat. 72 (2005), 93-100; English transl. in Theory Probab. Math. Statist. 72 (2006), 103-111. MR2168140 (2007b:60164)

3. A. V. Mel'nikov, Risk Analysis in Finance and Insurance, Chapman and Hall/CRC, 2004. MR.2013235 (2004i:91004)

4. A. V. Mel'nikov, Risk Management. Stochastic Analysis of Risks in Finance and Insurance, "Ankil", Moscow, 2001. (Russian)

5. A. V. Boikov, The Cramér-Lundberg model with stochastic premiums, Teor. Veroyatnost. i Primenen. 47 (2002), no. 3, 549-553; English transl. in Theory Probab. Appl. 47 (2003), no. 3, 489-493. MR.1975908 (2004d:60228)

6. N. Ikeda and S. Watanabe, Stochastic Differential Equations and Diffusion Processes, NorthHolland Publishing Co. and Kodansha, Ltd., Amsterdam-New York and Tokyo, 1981. MR.637061 (84b:60080)

Department of Mathematics, Faculty for Informatics, National Kyiv Mohyla University, Skovoroda Street, 2, Kyiv, 04070, Ukraine

E-mail address: mbratyk@ukr.net

Received 17/JUL/2006

Translated by N. SEMENOV 